\title{
BMJ Open Quality indicators for transition from paediatric to adult care for adolescents with chronic physical and mental illness: protocol for a systematic review
}

\author{
Katherine Bailey (D) , ${ }^{1,2}$ Stephanie Lee, ${ }^{3,4}$ Thomas de Los Reyes, ${ }^{5}$ Lisha Lo (D) ," \\ Jan Willem Gorter (D) , Alene Toulany ${ }^{1,2,8,9}$
}

To cite: Bailey K, Lee S, de Los Reyes T, et al. Quality indicators for transition from paediatric to adult care for adolescents with chronic physical and mental illness: protocol for a systematic review. BMJ Open 2021;11:e055194. doi:10.1136/ bmjopen-2021-055194

- Prepublication history and additional supplemental material for this paper are available online. To view these files, please visit the journal online (http://dx.doi.org/10.1136/ bmjopen-2021-055194)

Received 06 July 2021 Accepted 13 October 2021

Check for updates

(c) Author(s) (or their employer(s)) 2021. Re-use permitted under CC BY-NC. No commercial re-use. See rights and permissions. Published by BMJ.

For numbered affiliations see end of article.

Correspondence to

Dr Alene Toulany;

alene.toulany@sickkids.ca

\section{ABSTRACT}

Introduction Transition from paediatric to adult care is a complex process, which poses significant challenges for adolescents with chronic physical and mental illnesses. For many, transfer to adult care is associated with poor health and psychosocial outcomes. Quality indicators to evaluate transition to adult care are needed to benchmark and compare performance across conditions and health systems. This systematic review aims to identify quality indicators for successful transition to adult care which can be applied across chronic physical and/or mental illnesses. Methods Published literature will be searched using MEDLINE, Embase and CINHAL from earliest available date to July 2021. Grey literature will be searched using the Grey Matters tool. Using a set of inclusion/exclusion criteria, two independent reviewers will screen titles and abstracts, followed by full-text review. Disagreements will be resolved by a third reviewer. Study selection and data extraction will follow the Preferred Reporting Items for Systematic Reviews and Meta-Analyses Protocols. Study appraisal will be completed using the Appraisal of Guidelines for Research and Evaluation for Quality Indicators instrument. Extracted quality indicators will be categorised into a conceptual framework.

Ethics and dissemination Results from this review will offer novel insights into quality indicators that may be used to measure and evaluate transition success across conditions, which will be disseminated via a Canadian transition collaborative, workshops and peer-reviewed publication. Extracted quality indicators will be further prioritised in a Delphi study with patients, caregivers and providers. This is a critical step in developing a core set of metrics to evaluate transitions to adult care. Ethics approval is not required as this review will identify and synthesise findings from published literature.

PROSPERO registration number CRD42020198030.

\section{BACKGROUND}

Many adolescents with chronic physical and/or mental illnesses experience significant challenges during their transition from paediatric to adult care. ${ }^{1-7}$ They lose connection with the teams they trust, are required to make more decisions autonomously, receive less psychosocial support and often
Strengths and limitations of this study

- Quality indicators will only be included if developed through consensus-building methodology, and excluded if developed for evaluative studies and/or transition interventions.

- Quality indicators relating to all chronic illness populations, including physical, mental and developmental conditions, will be included.

- Critical appraisal of methods used for quality indicator development will be assessed via Appraisal of Guidelines for Research and Evaluation II instrument.

- A comprehensive grey literature search will be conducted to address publication bias.

- The search will be limited to literature in English language.

lack coordinated care between their multidisciplinary healthcare teams. ${ }^{189}$ Although there is variability across health jurisdictions, patients in Canada typically transfer to adult care between 16 and 19 years old. ${ }^{10}{ }^{11}$ This time of transfer often coincides with other critical milestones occurring in adolescence, making this a challenging period for many. ${ }^{12}$ Further, many youth feel they receive suboptimal care, are underprepared and dismissed during the process of transitioning to adult care. ${ }^{13-15}$ Potential patient safety risks associated with this transition include worsening disease states and complications, reduced adherence with treatment, emotional stress and delay or lost to follow-up with their adult provider. $^{14}{ }^{16-21}$

Despite the known gaps in transition care, there is no universally accepted definition of successful transition, or consensus on which metrics can be used to evaluate transition success overall. ${ }^{1722-24}$ Quality indicators are used to assess healthcare delivery, processes and outcomes to monitor and improve quality of care. ${ }^{25-27}$ They are developed using robust 
methodological approaches which incorporate high level evidence and input from experts. ${ }^{28}{ }^{29}$ Quality indicators are crucial for benchmarking patient, caregiver, provider and system-level performance to allow for meaningful evaluation and comparisons between hospitals, jurisdictions and health systems. ${ }^{30}$ Currently, there are no universally agreed on quality indicators in transition care which can be used for benchmarking across all chronic illnesses. Consequently, the lack of established universal metrics needed to improve quality of transition services limits advancement in the field.

Previous research has identified several indicators and outcomes of successful transition, such as continuity of care between paediatric and adult services, self-efficacy skills, disease-specific knowledge, treatment adherence and patient satisfaction with the transition process. ${ }^{10} 31-33$ However, many of these quality indicators have been limited to specific disease populations or specialties, hindering its generalisability and universal applicability across jurisdictions in Canada and beyond.

\section{OBJECTIVES AND RATIONALE}

The primary aims of this systematic review are to: (1) identify quality indicators of successful transition to adult care in adolescents with chronic illness, including physical, mental and developmental conditions and (2) categorise indicators into a conceptual framework. Given the limited literature integrating the diverse perspectives of all stakeholders, this review will include indicators proposed by patients, caregivers, clinicians and health system leaders. Quality indicators identified by this review will be prioritised in a Delphi study to develop consensus on metrics used to evaluate transition success.

\section{METHODS/DESIGN}

\section{Research question}

The primary research question was developed based on existing gaps in the literature:

1. What illness-specific and non-illness specific quality indicators have been developed via consensus-building methodology that define a successful transition to adult care based on patient, caregiver, provider or health system-level perspectives?

To answer this question, a systematic review was chosen to evaluate existing quality indicators for transition to adult care. Study will run from January 2021 to October 2021.

\section{Search strategy}

The literature search will be conducted using MEDLINE, Embase and CINAHL from earliest available date to July 2021. The search strategy will be developed with insights from a librarian and the research team. It will include a combination of two main concepts: (1) 'transition to adult care' and (2) 'benchmarking'. A detailed search strategy for MEDLINE is provided in online supplemental

\section{Title/Abstract Form $(n=10)$}

Two reviewers will independently pilot level 1

inclusion/exclusion criteria and record any changes, if necessary.

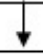

\section{Title/Abstract Form $(n=200)$}

Agreement between reviewers will be assessed to ensure kappa > 0.61 .

\section{Full-Text Review Form (n=10)}

Two reviewers will independently pilot level 2

inclusion/exclusion criteria and record any changes, if necessary.

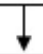

\section{Full-Text Review Form $(n=25)$}

Agreement between reviewers will be assessed to ensure kappa > 0.61 .

\section{$\downarrow$}

\section{Data Abstraction Form $(n=3)$}

Two reviewers will independently pilot the data abstraction form and record any changes, if necessary.

\section{1}

“Grey Matters" Framework for grey literature search (n=5)

Two reviewers will independently pilot search terms used in each database and establish number of results to be screened for each database.

\section{AGREE II-QI instrument (n=2)}

Two reviewers will independently pilot the AGREE II-QI instrument to assess its utility in the context of this review.

Figure 1 Summary of piloting protocol for systematic review. AGREE II-QI, Appraisal of Guidelines for Research and Evaluation II instrument for Quality Indicators.

appendix A. Titles and abstracts of articles obtained from the search will be exported to an Excel sheet for study selection.

\section{Study eligibility and selection}

A title/abstract study eligibility form will be developed by the research team, with involvement of a methodologist with expertise in conducting systematic reviews. Prior to screening articles, the form will be piloted by two independent reviewers (SL and TdLR) with 10 articles to ensure the inclusion/exclusion criteria are appropriate (piloting protocol for entire review is displayed in figure 1). Changes or clarifications to the study eligibility form will be made and reported in the final manuscript, if necessary. Following this, 200 papers will be screened to assess agreement between reviewers. Adjustments will be 
made until kappa $>0.61$ is achieved, indicating substantial agreement between reviewers. ${ }^{34}$

Study eligibility will be determined through two distinct steps: title/abstract screening (level 1) and full-text review (level 2). In level 1, studies will be included if they (1) focus on transition from paediatric to adult healthcare services and (2) use metrics or indicators to evaluate transition processes and outcomes. Studies will be excluded if they are not in English or are non-peer reviewed. Assessment of inclusion/exclusion criteria will be completed by two independent reviewers (SL and KB/TdLR). Decisions will be recorded using a study eligibility form administered via GoogleForms. Any disagreements will prompt re-evaluation by both reviewers, followed by resolution by a third reviewer (AT) if consensus cannot be achieved.

Studies included from level 1 will be read in full text and additional inclusion/exclusion criteria will be applied. Specifically, to improve content validity, studies will be included if quality indicators were developed using a robust consensus-building process (eg, Delphi method, prioritisation exercise, RAND/UCLA appropriateness methodology). Studies will be excluded if methodology used to develop quality indicators was not available, or if quality indicators were used to evaluate specific local transition interventions. Full-text review will be conducted by two independent reviewers (SL and $\mathrm{KB}$ ) and disagreements will be resolved by a third reviewer (AT). Intercoder agreement for both title/abstract and full-text screening will be measured using Cohen's kappa.

\section{Data extraction}

Data from included literature will be extracted using an abstraction tool developed on Microsoft Excel. The tool will include the following components: (1) study information (eg, title; author; year of publication; country; context/setting), (2) study methodology (eg, study design, population, illness/condition) and (3) quality indicator information (eg, quality indicator description; category of indicator; perspective of indicator). A detailed data abstraction form is included in table 1 . The data abstraction tool will be piloted for the first three studies and changes will be made accordingly. Data will be extracted by two independent reviewers (SL and $\mathrm{KB}$ ) using two separate data abstraction forms. The information extracted will then be combined into one Excel sheet. Disagreements between reviewers will be resolved by a third reviewer (AT). All extracted data will be reported on in accordance with Preferred Reporting Items for Systematic Reviews and Meta-Analyses Protocol (PRISMA-P) guidelines. ${ }^{35}$

\section{Grey literature search}

Grey literature will be searched from July 2021 to earliest available date following the Canadian Agency for Drugs and Technology in Health 'Grey Matters' framework. ${ }^{36}$ Search terms will include 'quality indicators' and 'transition to adult care', and will be piloted for the first five databases included in the Grey Matters framework. Additionally, the National Institute for Health and Care Excellence, National Quality Forum and Agency for Health Research and Quality databases will be hand-searched with more extensive search terms for reports and/or guidelines identifying indicators for transition. Two separate reviewers will complete the grey literature search and record findings in the checklist provided by grey matters (KB and LL). The data abstraction form will be modified for the grey literature search.

\section{Study appraisal}

Studies will be critically appraised using a modified version of the Appraisal of Guidelines for Research and

Table 1 Information to be extracted from studies included in systematic review

\begin{tabular}{ll}
\hline Category & Data to be extracted \\
Study information & Author(s) \\
& Title of article \\
& Year \\
Study methodology & Country of origin \\
& Study design \\
& Population \\
& Sample size \\
& Panel characteristics (ie, expert vs patient panels; paediatric vs adult providers) \\
& Data collection method \\
& Clinical context/disease \\
& Description of indicator \\
& Indicator category (illness-specific vs non-illness specific) \\
Quality indicators & Indicator level (ie, patient, caregiver, provider, health system) \\
& Classification according to Donabedian model (ie, structure, process, outcome) \\
& effective, efficient, safe, timely, equitable) \\
& Information for measurement of quality indicator (eg, instructions for use, frequency/interval of \\
& measurement, tools for measurement) \\
& Associated evidence for quality indicator \\
\hline &
\end{tabular}


Evaluation II instrument for Quality Indicators (AGREE II-QI) ${ }^{37}$ The AGREE II-QI tool has been previously used to evaluate the development of quality indicators. ${ }^{38} 39$ Evaluation of the process used to develop quality indicators will be based on six domains: scope and purpose, stakeholder involvement, rigour of development, clarity of presentation, applicability and editorial independence. Degree to which quality indicators meet domain items will be rated on a 7-point Likert scale (1='strongly disagree'; $7=$ 'strongly agree'). The AGREE II-QI instrument will be piloted for the first two included studies to assess utility of the tool in the context of this systematic review. Two independent reviewers will complete the critical appraisal for all included studies (KB and LL). Ratings will be compiled into a Microsoft Excel spreadsheet and domain scores will calculated in accordance with the scoring system outlined in the AGREE II user manual. ${ }^{32}$

\section{Summarising results}

Study characteristics recorded in the data extraction form will be presented in a table, following PRISMA-P guidelines. Extracted quality indicators will be organised into a conceptual framework with illness-specific and non-illness specific categories. Indicators will be further subcategorised into (1) patient, (2) caregiver, (3) provider and (4) health system-level. The Donabedian model and the Institute of Medicine (IOM) Domains of Healthcare Quality are two well-established quality measurement frameworks which will be used to classify identified quality indicators. ${ }^{26} 40-42$ According to the Donabedian model, quality indicators will be classified as structure (ie, metrics assessing the characteristics of the care setting, such as human resources, models of care and availability of services), process (ie, metrics assessing the delivery of care, including patient education, counselling, diagnosis and treatments) or outcome (ie, metrics assessing the effect of healthcare services on the health of the patients and is frequently). ${ }^{26} 404143$ Using the IOM Domains of Healthcare Quality, quality indicators will be classified as patient-centred (ie, ensures patient values guide clinical decisions), equitable (ie, provides high-quality care to all, regardless of personal characteristics), efficient (ie, avoids waste), effective (ie, practices evidence-based medicine), safe (ie, avoids harm) and timely (ie, reduces wait times and delays in receiving care). ${ }^{42}{ }^{44}$ Classifying quality indicators by these frameworks will allow us to identify any potential gaps in measurement (ie, identify any missing categories of indicators, disproportionate number of indicators in certain categories). It is expected this framework will evolve as indicators are identified through the review, and changes will be made accordingly.

\section{Patient and public involvement}

The principal investigator of this systematic review, AT, is cochair of a Canada-wide Quality Indicators in Transition Subcommittee of Children Healthcare Canada's Health Hub in Transition. ${ }^{10}$ This network was founded in 2019 and aims to collaborate with stakeholders across the continuum of healthcare delivery to coordinate the services provided during transition to adult care. Committee membership includes representative national stakeholders, including patients, caregivers, health leaders and multidisciplinary healthcare providers with diverse geographical representation and cross-condition expertise (eg, physical, mental and developmental conditions). The Health Hub in Transition is driven by conducting research informed by the needs of patients and caregivers. ${ }^{10}$ Throughout the development of this systematic review, the research team has been continually seeking input from committee members on study objectives and knowledge translation activities. As results are identified, the research team will continue to consult the committee to refine the conceptual framework.

\section{DISCUSSION}

This review will identify quality indicators for transition to adult care for adolescents with chronic physical and/or mental illnesses. Consensus on which quality indicators are needed to measure transition success is necessary to ensure comprehensive evaluation of patient and systemlevel outcomes.

Few studies have identified which measures of transition success matter to adolescents with chronic illnesses. A qualitative meta-synthesis highlighted youth expectations of successful transition, including transition readiness, transition education and independence. ${ }^{45}$ While research has suggested adolescents and their caregivers have different priorities and needs from their clinicians, the majority of existing quality indicators have been proposed exclusively by healthcare providers. ${ }^{12}$ 46-48 $\mathrm{A}$ recent Delphi survey engaged patients, caregivers and healthcare providers to identify core components of successful transition from paediatric to adult mental health services. ${ }^{49}$ However, these findings cannot be generalised to reflect the priorities of patients with chronic physical illnesses and their caregivers. Further, a study found indicators of transition success (eg, lost to follow-up, trust with adult provider) were partially related to patient-reported outcomes (eg, adherence to therapeutic regimen, disease-related selfefficacy).$^{50}$ Without incorporating patient and caregiver perspectives in the development of quality indicators, it is difficult for health leaders, administrators and clinicians to benchmark, evaluate or compare transition processes and outcomes across conditions and jurisdictions.

Quality indicators in transition need to reflect the complexity of patient care needs, their strengths and capture both clinical and process outcomes. Social determinants of health, patient-reported outcomes and health literacy will also be prioritised within the framework we build based on the available evidence. We hope to work alongside health leaders and administrators to elevate the transition platform and identify and leverage funding opportunities to support needed transition work. In addition, our review will help inform more specificity and a 
minimum standard required for transition to adult care among healthcare accreditation agencies.

Although this systematic review will highlight the perspectives of key stakeholders in transition to adult care, there remain potential limitations to this approach. Notably, limiting inclusion of quality indicators to development through consensus-building methods may reduce the scope of indicators included in the review. Several studies, including quality improvement initiatives and evaluation of transition interventions, have developed quality indicators via non-consensus building methods (eg, literature review, one-on-one interviews) and will not be captured in thissystemic review. Our review is only limited to literature in English language.

\section{ETHICS AND DISSEMINATION}

Ethics approval is not required by our institution for this study as this systematic review will synthesise findings from published literature. Results of this systematic review will be submitted for publication in a peer-reviewed journal. Additionally, abstracts will be submitted for oral and/or poster presentations. Findings will be disseminated to national stakeholders, including healthcare providers, patients and caregivers, through a Canada-wide collaborative, Health Hub in Transition, to gain insights into next steps and knowledge translation activities.

Quality indicators identified will be prioritised in a Delphi process with healthcare providers, patients and caregivers. Results will be used to develop a set of universal quality indicators for assessing transition. It is hoped this review will contribute to the development of best practices for measuring healthcare performance and provide a framework for evaluating quality improvement initiatives in transition to adult care.

\section{Author affiliations \\ ${ }^{1}$ Temerty Faculty of Medicine, University of Toronto, Toronto, Ontario, Canada ${ }^{2}$ Institute of Health Policy Management and Evaluation, University of Toronto, Toronto, Ontario, Canada \\ ${ }^{3}$ Department of Medicine, The Royal Children's Hospital Melbourne, Parkville, Victoria, Australia \\ ${ }^{4}$ Department of Adolescent Medicine, Monash Children's Hospital, Clayton, New South Wales, Australia \\ ${ }^{5}$ Department of Urology, The Hospital for Sick Children, Toronto, Ontario, Canada \\ ${ }^{6}$ Centre for Quality Improvement and Patient Safety, University of Toronto, Toronto, Ontario, Canada \\ ${ }^{7}$ CanChild Centre for Childhood Disability Research, Department of Pediatrics, McMaster University, Hamilton, Ontario, Canada \\ ${ }^{8}$ Division of Adolescent Medicine, The Hospital for Sick Children, Toronto, Ontario, Canada \\ ${ }^{9}$ Department of Pediatrics, University of Toronto, Toronto, Ontario, Canada}

\section{Twitter Jan Willem Gorter @Dr_Gorter}

Acknowledgements The authors thank Jacklynn Pidduck, cochair of the Transition Quality Indicators subcommittee, subset of the Children Healthcare Canada's Health Hub in Transition. The authors also wish to thank other members of the committee, including Dayle McCauley, Heather Buckingham, Dr Gail Andrews, Dr Michelle Batthish, Dr Karen Beattie, Dr Lorraine Bell, Dr Emilie Dionne, Danijela Grahovac, Dr Yani Hamdani, Amanda Higgins, Ariana Wong, Brooke Allemang, Dr Kristin Cleverley, Jaime Winkler, Meghan Patton, Dilshad Kassam-Lillani, Sarah Sheffe, Christina Gilman, and Alice Soper for their insights into the development of this protocol. We also thank Ms. Quenby Mahood, reference librarian at the Hospital for Sick Children, for developing the search strategy for this review. Dr Gorter is the inaugural chair of the Health Hub in Transition and holds the Scotiabank Chair in Child Health Research.

Contributors AT and JWG conceptualised the design and methods of this systematic review, revised the manuscript and approved the final manuscript as submitted. KB synthesised the literature, drafted the initial manuscript, revised the manuscript and approved the final manuscript as submitted. SL and TdLR synthesised the literature, revised the manuscript and approved the final manuscript as submitted. LL provided methodological expertise, revised the manuscript and approved the final manuscript as submitted. All authors provided input and guidance on study design. All authors approved the final manuscript as submitted and agree to be accountable for all aspects of this systematic review.

Funding The authors have not declared a specific grant for this research from any funding agency in the public, commercial or not-for-profit sectors.

Competing interests None declared.

Patient consent for publication Not applicable.

Provenance and peer review Not commissioned; externally peer reviewed.

Supplemental material This content has been supplied by the author(s). It has not been vetted by BMJ Publishing Group Limited (BMJ) and may not have been peer-reviewed. Any opinions or recommendations discussed are solely those of the author(s) and are not endorsed by BMJ. BMJ disclaims all liability and responsibility arising from any reliance placed on the content. Where the content includes any translated material, BMJ does not warrant the accuracy and reliability of the translations (including but not limited to local regulations, clinical guidelines, terminology, drug names and drug dosages), and is not responsible for any error and/or omissions arising from translation and adaptation or otherwise.

Open access This is an open access article distributed in accordance with the Creative Commons Attribution Non Commercial (CC BY-NC 4.0) license, which permits others to distribute, remix, adapt, build upon this work non-commercially, and license their derivative works on different terms, provided the original work is properly cited, appropriate credit is given, any changes made indicated, and the use is non-commercial. See: http://creativecommons.org/licenses/by-nc/4.0/.

\section{ORCID iDs}

Katherine Bailey http://orcid.org/0000-0001-6486-4818

Lisha Lo http://orcid.org/0000-0002-3875-4372

Jan Willem Gorter http://orcid.org/0000-0002-3012-2119

\section{REFERENCES}

1 Viner R. Transition from paediatric to adult care. Bridging the gaps or passing the buck? Arch Dis Child 1999;81:271-5.

2 Camfield P, Camfield C. Transition to adult care for children with chronic neurological disorders. Ann Neurol 2011;69:437-44.

3 Kirk S. Transitions in the lives of young people with complex healthcare needs. Child Care Health Dev 2008;34:567-75.

4 O'Connell B, Bailey S, Pearce J. Straddling the pathway from paediatrician to mainstream health care: transition issues experienced in disability care. Aust $J$ Rural Health 2003;11:57-63.

5 Kaufman M, Pinzon J. Transition to adult care for youth with special health care needs. Paediatr Child Health 2007;12:785-8.

6 Islam Z, Ford T, Kramer T, et al. Mind how you cross the gap! Outcomes for young people who failed to make the transition from child to adult services: the TRACK study. BJPsych Bull 2016;40:142-8.

7 Paul M, Ford T, Kramer T, et al. Transfers and transitions between child and adult mental health services. Br J Psychiatry Suppl 2013;54:s36-40.

8 High rate of graft loss in pediatric kidney recipients who transition to an adult transplant program; roles of non-adherence and late referral. Am J Transplant.

9 Watson AR, Harden P, Ferris M, et al. Transition from pediatric to adult renal services: a consensus statement by the International Society of nephrology (ISN) and the International pediatric nephrology association (IPNA). Pediatr Nephrol 2011;26:1753-7.

10 Transition to adulthood: health hub in transition, 2021. Available: https://www.transitionhub.ca/ [Accessed 23 Jun 2021].

11 Canadian Association of Pediatric Health Centres (CAPHC), National Transitions Community of Practice. A guideline for transition from paediatric to adult health care for youth with special health care needs: a national approach [Guideline], 2016. Available: http://ken. 
caphc.org/xwiki/bin/view/Transitioning+from+Paediatric+to+Adult+ Care/A+Guideline+for+Transition+from+Paediatric+to+Adult+Care [Accessed 23 Jun 2021].

12 Sun HL, Breakey VR, Straatman L, et al. Outcomes indicators and processes in transitional care in adolescents with haemophilia: a Delphi survey of Canadian haemophilia care providers. Haemophilia 2019;25:296-305.

13 Kelly A, Niddrie F, Tunnicliffe DJ, et al. Patients' attitudes and experiences of transition from paediatric to adult healthcare in rheumatology: a qualitative systematic review. Rheumatology 2020;59:3737-50.

14 Appleton R, Connell C, Fairclough E, et al. Outcomes of young people who reach the transition boundary of child and adolescent mental health services: a systematic review. Eur Child Adolesc Psychiatry 2019;28:1431-46.

15 Crawford K, Wilson C, Low JK, et al. Transitioning adolescents to adult nephrology care: a systematic review of the experiences of adolescents, parents, and health professionals. Pediatr Nephrol 2020;35:555-67.

16 Annunziato RA, Baisley MC, Arrato N, et al. Strangers headed to a strange land? A pilot study of using a transition coordinator to improve transfer from pediatric to adult services. $J$ Pediatr 2013;163:1628-33.

17 Watson R, Parr JR, Joyce C, et al. Models of transitional care for young people with complex health needs: a scoping review. Child Care Health Dev 2011;37:780-91.

18 Knauth Meadows A, Bosco V, Tong E, et al. Transition and transfer from pediatric to adult care of young adults with complex congenita heart disease. Curr Cardiol Rep 2009;11:291-7.

19 Bohun CM, Woods P, Winter C, et al. Challenges of intra-institutional transfer of care from paediatric to adult congenital cardiology: the need for retention as well as transition. Cardiol Young 2016;26:327-33.

20 Luque Ramos A, Hoffmann F, Albrecht K, et al. Transition to adult rheumatology care is necessary to maintain DMARD therapy in young people with juvenile idiopathic arthritis. Semin Arthritis Rheum 2017;47:269-75.

21 Ritchwood TD, Malo V, Jones C, et al. Healthcare retention and clinical outcomes among adolescents living with HIV after transition from pediatric to adult care: a systematic review. BMC Public Health 2020;20:1195.

22 Davis AM, Brown RF, Taylor JL, et al. Transition care for children with special health care needs. Pediatrics 2014;134:900-8.

23 Fletcher-Johnston M, Marshall SK, Straatman L. Healthcare transitions for adolescents with chronic life-threatening conditions using a Delphi method to identify research priorities for clinicians and academics in Canada. Child Care Health Dev 2011;37:875-82.

24 Fair CD, Sullivan K, Gatto A. Indicators of transition success for youth living with HIV: perspectives of pediatric and adult infectious disease care providers. AIDS Care 2011;23:965-70.

25 Mainz J, Krog BR, Bjørnshave B, et al. Nationwide continuous quality improvement using clinical indicators: the Danish national indicator project. Int J Qual Health Care 2004;16:i45-50.

26 Donabedian A. Exploratings in quality assessment and monitoring definition of quality and approaches to its assessment. Ann Arbor 1980.

27 Brook RH, McGlynn EA, Cleary PD. Measuring quality of care. N Engl J Med Overseas Ed 1996;335:966-70.

28 Hearnshaw HM, Harker RM, Cheater FM, et al. Expert consensus on the desirable characteristics of review criteria for improvement of health care quality. Qual Health Care 2001;10:173-8.

29 Mainz J. Defining and classifying clinical indicators for quality improvement. Int J Qual Health Care 2003;15:523-30.
30 Hermann RC, Mattke S, Somekh D, et al. Quality indicators for international benchmarking of mental health care. Int J Qual Health Care 2006;18:31-8.

31 Sobota AE, Shah N, Mack JW. Development of quality indicators for transition from pediatric to adult care in sickle cell disease: a modified Delphi survey of adult providers. Pediatr Blood Cancer 2017;64:e26374. doi:10.1002/pbc.26374

32 van den Brink G, van Gaalen MAC, de Ridder L, et al. Health care transition outcomes in inflammatory bowel disease: a multinational Delphi study. J Crohns Colitis 2019;13:1163-72.

33 Erős A, Soós A, Hegyi P, et al. Spotlight on transition in patients with inflammatory bowel disease: a systematic review. Inflamm Bowel Dis 2020;26:331-46.

$34 \mathrm{McHugh} \mathrm{ML}$. Interrater reliability: the kappa statistic. Biochem Med 2012;22:276-82.

35 Moher D, Shamseer L, Clarke M, et al. Preferred reporting items for systematic review and meta-analysis protocols (PRISMA-P) 2015 statement. Syst Rev 2015;4:1.

36 Canadian Agency for Drugs and Technologies in Health. Grey matters: a practical search tool for evidence-based medicine, 2018. Available: https://www.cadth.ca/resources/finding-evidence/greymatters [Accessed 23 Jun 2021].

37 Brouwers MC, Kho ME, Browman GP, et al. AGREE II: advancing guideline development, reporting and evaluation in health care. CMAJ 2010;182:E839-42.

38 Abrahamyan L, Boom N, Donovan LR, et al. An international environmental scan of quality indicators for cardiovascular care. Can J Cardiol 2012;28:110-8.

39 Cooper M, Rouhi A, Barber CEH. A systematic review of quality measures for inflammatory arthritis. J Rheumatol 2018;45:274-83.

40 Donabedian A. Evaluating the quality of medical care. 1966. Milbank Q 2005;83:691-729.

41 Donabedian A. An introduction to quality assurance in health care 2002.

42 lo M. Performance measurement: accelerating improvement. Washington, DC: The National Academies Press, 2006.

43 Donabedian A. The quality of care. JAMA 1988;260:1743-8.

44 Institute of Medicine Committee on Quality of Health Care in A. Crossing the quality chasm: a new health system for the 21st century. Washington, DC: National Academies Press, 2001.

45 Varty M, Speller-Brown B, Phillips L, et al. Youths' experiences of transition from pediatric to adult care: an updated qualitative Metasynthesis. J Pediatr Nurs 2020;55:201-10.

46 Akre C, Suris J-C, Belot A, et al. Building a transitional care checklist in rheumatology: a Delphi-like survey. Joint Bone Spine 2018;85:435-40.

47 Broad KL, Sandhu VK, Sunderji N, et al. Youth experiences of transition from child mental health services to adult mental health services: a qualitative thematic synthesis. BMC Psychiatry 2017:17:380.

48 Suris J-C, Akre C. Key elements for, and indicators of, a successful transition: an international Delphi study. $J$ Adolesc Health 2015;56:612-8.

49 Cleverley K, McCann E, O'Brien D. Prioritizing core components of successful transitions from child to adult mental health care: a national Delphi survey with youth, caregivers, and health professionals. Eur Child Adolesc Psychiatry 2021:1. doi:10.1007/s00787-021-01806-6

50 Sattoe JNT, Hilberink SR, van Staa A. How to define successful transition? An exploration of consensus indicators and outcomes in young adults with chronic conditions. Child Care Health Dev 2017:43:768-73. 